\title{
Simulation Algorithm to Determine the Number of Un-Served Calls when Transferring the Signal in Broadband Cellular Networks
}

\section{E. Otsetova-Dudin, I. Kurtev, S. Siarova}

Key words: Simulation models; simulation algorithm; broadband handover; rare events.

\begin{abstract}
Planning and design of cellular networks is associated with the creation of traffic models for the transfer of signal using schemes with priorities within which assess the probability parameters of quality of service is performed. The study of these parameters is associated with the creation of a simulation model and an algorithm of broadband handover.
\end{abstract}

\section{Introduction}

In urban mobile cellular environments the signal transfer has a significant impact on the system performance. Therefore, it is necessary to develop traffic models for broadband handover in order to improve the parameters of quality of service $(Q o S)$, by reducing the number of unserviced calls. The need to ensure constant connectivity of mobile users with high $Q o S$ in today's mobile networks require the study of rare events $[1,7,10]$.

Handover is the process of changing the channel (frequency, time slot, spreading code, or combination of them) associated with the current connection while a call is in progress [1].

Handover often is initiated either by crossing a cell boundary or by deterioration in quality of the signal in the current channel [2]. In broadband wireless networks especially when the cell size becomes relatively small, the handover procedure has a significant impact on system performance [14].

There are strict requirements for $Q o S$ parameters in broadband handover. These parameters are probabilistic in nature and should not exceed a value greater $10^{-9}$, which qualifies them as rare events [1,2]. The study of probability parameters of quality of service requires development of simulation models and algorithms for signal transfer through schemes with priorities $[5,8]$.

This paper presents a simulation algorithm that determines the number of unserviced calls due to non-availability of a free channel at the base station and handover queue overflow. A scheme with two queues, providing three levels of priority service is used.

\section{Rare Events Simulation}

In order to estimate the probability of rare event occurrence it is necessary that the event appears repeatedly. This requires the use of different methods to accelerate the simulation [5].

Accelerating Simulation Techniques transform a familiar model into a new, which more quickly and accurately assess the studied parameters [3]. Some of the most commonly used methods to accelerate the simulations are:

$\checkmark$ Methods that reduce dispersion by simple carbohydrates random numbers - Common Random Numbers $(C R N)$, Controlled Variables $(\mathrm{CV})$ and Indirect Estimation (IE);

$\checkmark$ Rare Event Simulation Methods (RES), by generating a pseudo-random numbers - a Monte Carlo method $(M C)$, branching trajectory models Splitting, generation of significant randomization sample Importance Sampling (IS) [12]. In the $I S$ method conditional probability of a rare event occurrence is replaced by the conditional probability of a new transfer function in which the observed event occurs more often $[4,7,13]$ (figure 1).

This simulation approach requires generating independent samples with a certain differential distribution law of each iteration (figure 1) [5]. For evaluation of the probability of rare events by Importance Sampling method the weight function $L(x)$ is used $[3,6]$

(1) $L(x)=\left\{\begin{array}{l}\frac{f(x)}{f^{*}(x)}, f(x) \neq 0, \\ 0\end{array}\right.$

where function $f(x)$ is differential distribution of the tested function and $f^{*}(x)$ is the new function.

(2) $\int_{+\infty}^{-\infty} f(x) f^{*}(x) d x=1$.

For $I S$ approach is typical that a new function $f^{*}(x)$ is selected so that for each value of $\mathrm{x}$ by distribution $f(x)$ the following expression is valid:

(3) $P(Y<t)=F(t)=\int_{-\infty}^{t} f(y) d y=\int_{-\infty}^{t} \frac{f(x)}{f^{*}(x)} f^{*}(x) d x$.

The evaluation of the a rare event occurrence is de- 


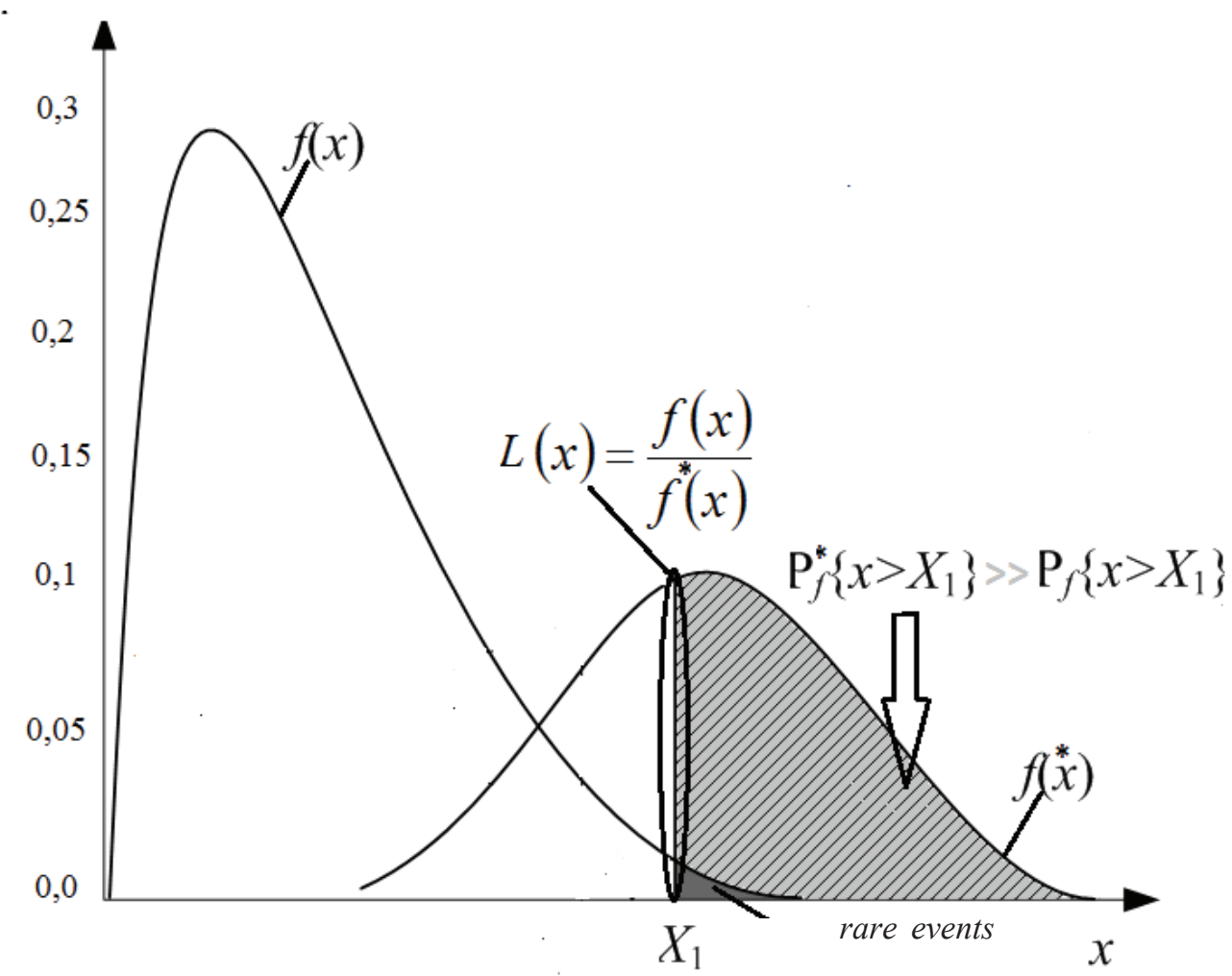

Figure 1. Importance Sampling Simulation Technique

termined so that it remains uncorrelated when choosing $N$ independent samples from the distribution $f^{*}(x)$.

The probability of rare event occurrence is expressed by the degree of similarity, and is difined as

(4) $\hat{\gamma}^{(I S)}=\frac{1}{N} \sum_{n=1}^{N} g\left(x_{n}\right) L\left(x_{n}\right)$,

where $g(x)$ is the observed event. There is a limitation for the selection of the new function $-f^{*}(x)>0$ for every $x$, where $g(x) f(x) \neq 0[6,12]$.

The effectiveness of simulation result depends on proper selection of new distribution. There is a possibility to increase the dispertion when a wrong selection of distribution is made. There is a possibility that dispersion can reach a value higher than the dispersion as a result of Monte Carlo approach application. Which in turn makes use of a method for generating a significant randomisation unnecessary $[3,5,6]$.

A review of the scientific literature found that Monte Carlo method is not effective when the probability of events occurrence is smaller than $10^{-6}[4]$.

A major disadvantage of the Splitting and Importance Sampling methods is a strong reduction of areas in which we expected occurrence of rare events [5]. This requires the development of new methods for rare events similation.

\section{Simulation Model of Handover Implemented with Two Queues}

Losses from the transfer of signals in broadband cel- lular radio networks and new requirements for improving the quality of service required the development of new traffic models and algorithms. Rare event simulations allow the evaluation of the probability parameters of the service quality in the handover, such as the probability of blocking of the channel at the base station and the handover queue overflow. Based on [1,7] there is a new methodology for $Q o S$ assessment of handover in broadband cellular networks presented in [11]. One stage of this methodology is the implementation of the simulation model and algorithm for determining the number of unserviced calls in handover by priorities schemes.

Based on the traffic models of the signal transfer, discussed in $[8,9,10,14]$ the simulation model of handover with three priority is presented (figure 2).

The priority level of the selected simulation model are presented through distribution channels in the base station for new coming calls, for data calls waiting to be served in the queue $q_{\mathrm{N}}$, for voice calls $q_{\mathrm{R}}$ and by setting the maximum lengths of the handover queues $M_{\mathrm{N}}$ and $M_{\mathrm{R}}$. With highest priority are voice calls in queue $q_{\mathrm{R}}$, followed by data calls waiting to be served in the queue. The new coming calls in the channels of the base station are with the lowest priority $[8,9]$.

The main objective of this simulation model is to reduce losses and delay in service of voice calls by a scheme with two queues. In this model, input parameters are:

$\checkmark$ Channels for new coming calls $N_{\mathrm{C}}$ and $N_{\mathrm{N}}$ channels for data calls in the handover queue $q_{\mathrm{N}}$;

$\checkmark$ The total number of channels in the base station $N$; 


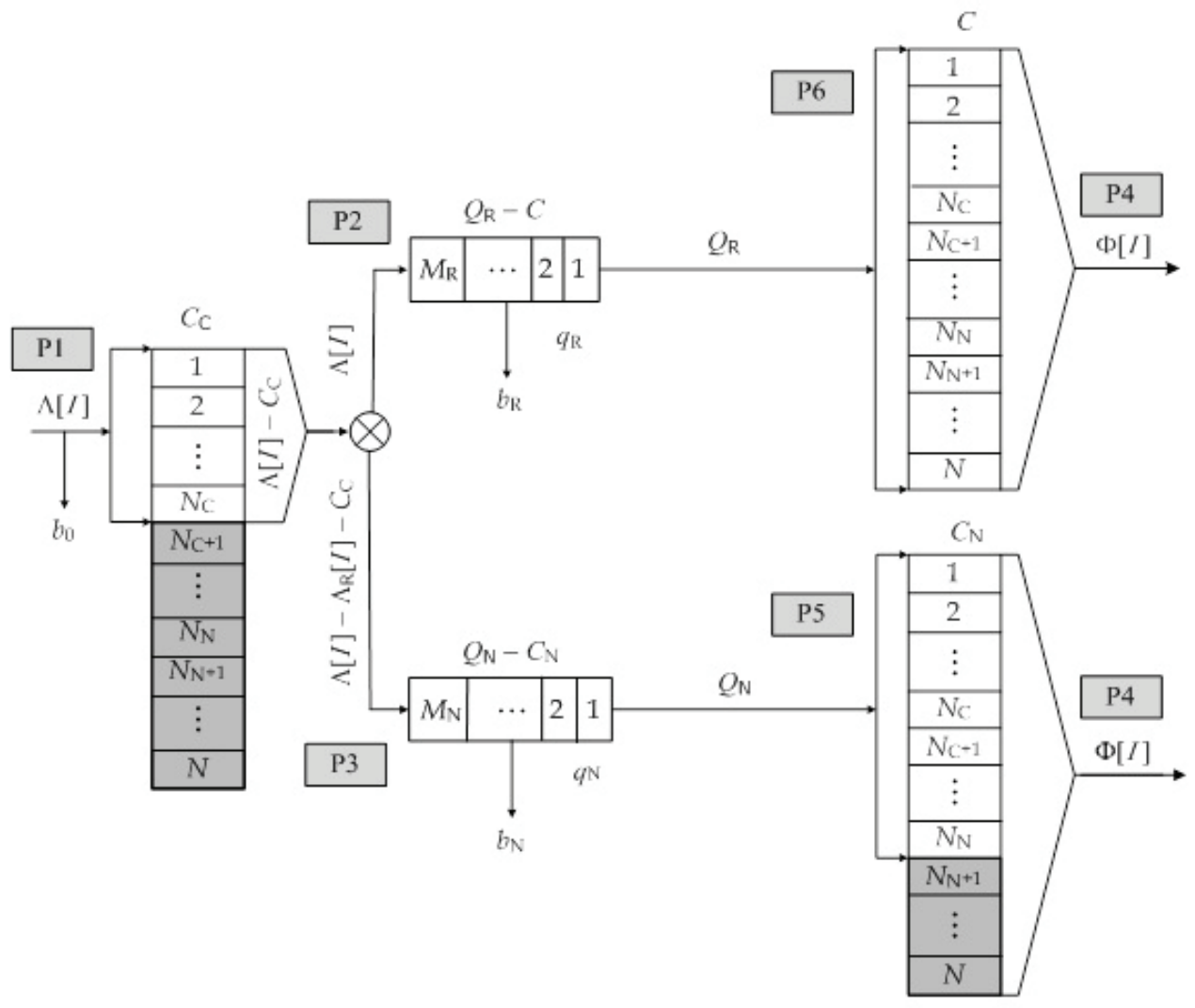

Figure 2. Simulation model of handover implemented with two queues

for data calls $M_{\mathrm{N}}$;

$\checkmark$ The lengths of the queues for voice calls $M_{\mathrm{R}}$ and

$\checkmark$ The number of iterations $n$.

The simulation model presented in figure 2 used three random number generator:

Two generators that determine static traffic calls for incoming flow and voice calls flow which have not found a free channel and have been forwarded to the queue $q R$. For this purpose, different distribution laws are applied Poisson distribution, Gaussian distribution, Logarithmic distribution and Uniform distribution. The generated value for voice calls have to perform the inequality: $\Lambda_{\mathrm{R}}[I]<\left(\Lambda[I]-N_{\mathrm{C}}\right)$ for each iteration.

The third generator is used to set the number of channels that will be served at each iteration by using exponentially, Uniform and Gaussian distribution.

The simulation model presented in figure 2 includes six major steps (designated by $\mathrm{P}$ ).

First step P1- new coming calls occupy the channels from 1 to $N_{\mathrm{C}}$. In cases when the number of these calls is greater than the channels in the base station the number of voice calls which didn't find free channels generates a value of $\left(\Lambda[I]-C_{\mathrm{C}}[I]\right)$.

On step P2 the voice calls occupy the free channels in the handover queue $q_{\mathrm{R}}$, data calls occupy the free channels in the queue $q_{\mathrm{N}}$, where they wait to be served (step P3).

During the step P4 servicing of the busy channels is done. For this purpose a random number generator that defines the number of the served channels $\Phi[\mathrm{I}]$ with selected distribution is used.

On step P5 the data calls from handover queue $q_{\mathrm{N}}$ occupy the free channels in the base station from 1 to $N_{\mathrm{N}}$ with service discipline First-Come, First-Served (FCFS).

The voice calls waiting to be served in the handover queue $q_{\mathrm{R}}$, can occupy any free channel from 1 to $N$ (step P6).

The output parameters of the simulation model in figure 2 present losses occurred due to:

$\checkmark$ Blocking of the new coming calls in the channels of the base station and overflow queues of handover.

$\checkmark$ Overflow of the handover queues.

The queue overflow $q_{\mathrm{R}}$ appear in cases where the maximum queue length $M_{\mathrm{R}}$ is less than the number of generated voice calls, and the queue overflow for data traffic appear when the queue length for data traffic fulfill the condition: $M_{\mathrm{N}}<\left(\left(\Lambda[I]-N_{\mathrm{C}}\right)-\Lambda_{\mathrm{R}}[I]\right)$.

Block calls newcomers in the channels of the base station occurs at a capacity filled tails in handover and filled channels in the base station. For the correct operation of this model is necessary $N_{\mathrm{C}} \leq N_{\mathrm{N}} \leq N$. In the simulation algorithm the following dimensionless, operating parameters are defined:

$N_{\mathrm{C}} /\left(N-N_{\mathrm{N}}\right)$ - share of channels reserved for new coming calls, for data packets and voice calls in handover queues $q_{\mathrm{N}}$ and $q_{\mathrm{R}}$;

$M_{\mathrm{R}} / N$ - the ratio between the maximum queue length for voice calls and the total number of channels 


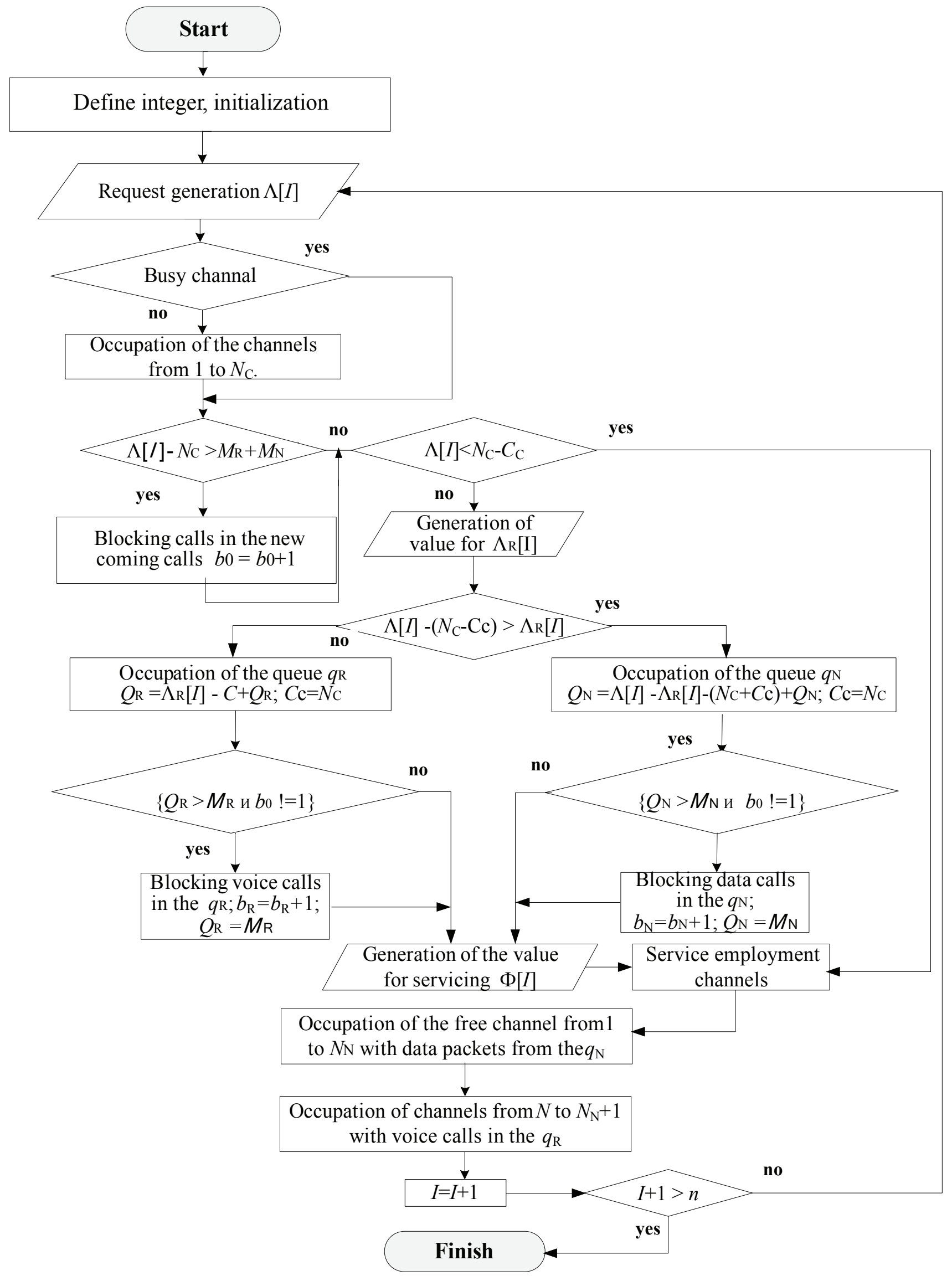

Figure 3. Simulation Algorithm 
in the base station;

$M_{\mathrm{N}} / \mathrm{N}$ - the ratio between the maximum queue length for data traffic and the total number of channels in the base station.

\section{The Simulation Algorithm for Determining the Number of Unserviced Calls}

Based on [7,8] a simulation algorithm for determining the number of unserviced calls is developed. The algorithm is represented by a block diagram in figure 3 .

We used the following variables and arrays:

$I$ - iterations;

Number of incoming calls $\Lambda[I]$;

The number of voice calls in handover queue $q_{\mathrm{R}}-$ $\Lambda_{\mathrm{R}}[I]$

The number of busy channels with new coming calls $-C_{\mathrm{C}}$;

The number of busy channels with data packets $-C_{\mathrm{N}}$; The number of busy channels in the base station $C$; Loan queues for voice calls $Q_{\mathrm{R}}, Q_{\mathrm{N}}$ in the queue $q_{\mathrm{N}}$; The number of blocking calls in the channels of the base station $b_{0}$;

Overflows in queue for data packets $b_{\mathrm{N}}$ and in queue $q_{\mathrm{R}}-b_{\mathrm{R}}$;

The generated sample size $n$ [9].

A block scheme of simulation algorithm for determining the number of unserviced calls (figure 3) can be represented by the following steps:

Step 1: Initialization of the system parameters $-N_{\mathrm{C}}$, $N_{\mathrm{N}}, N, M_{\mathrm{N}}, M_{\mathrm{R}}, I, Q_{\mathrm{R}}, Q_{\mathrm{N}}, C_{\mathrm{C}}, C, C_{\mathrm{N}}, b_{0}, b_{\mathrm{N}}, b_{\mathrm{N}}, n$ and arrays $\Lambda[I], \Lambda_{\mathrm{R}}[I], \Phi[I]$.

Step 2: Selection of the distribution laws for: incoming calls $\Lambda\left[I\right.$, voice calls $\Lambda_{\mathrm{R}}[I]$ and servicing of the busy channels in the base station $\Phi[I]$.

Step 3: Generation of values for input calls $\Lambda[I]$.

Step 4: Check the existence of free channels in the base station: in $\Lambda[I]<C_{C}$, a transition to Step 14 .

Step 5: Occupation of the channels in the base station with new coming calls with current value $C_{C}$.

Step 6: Check the existence of blocked new coming calls: if $\Lambda[I]>N_{\mathrm{C}}+M_{\mathrm{N}}+M_{\mathrm{R}}$, then count the number of blocked new coming calls $b_{0}=b_{0}+1$ as $\Lambda[I]=N_{\mathrm{C}}+\left(M_{\mathrm{N}}+M_{\mathrm{R}}\right)$.

Step 7: Check the existence of free channels: if $\Lambda[I] \leq N_{\mathrm{C}}-C_{C}$, then transition to step 14 .

Step 8: Generation of value for $\Lambda_{\mathrm{R}}[I]$, if $\Lambda_{\mathrm{R}}[I]<\Lambda\left[I-C_{\mathrm{C}}\right.$.

Step 9: Occupation of a queue $q_{\mathrm{R}}$ with current value $Q_{\mathrm{R}}=\Lambda[I]-\Lambda_{\mathrm{R}}[\mathrm{I}]-\left(N_{\mathrm{C}}+C_{\mathrm{C}}\right)+Q$, as $C_{C}=N_{\mathrm{C}}$.

Step 10: Check for queue overflow $q_{\mathrm{R}}$ : if $Q_{\mathrm{R}}>M_{\mathrm{R}}$ and $b_{0} \neq 1$, then count the number of queue overflows $b_{\mathrm{R}}=b_{\mathrm{R}}$ +1 , as $Q_{\mathrm{R}}=M_{\mathrm{R}}$.

Step 11: Check for the existence of data traffic to queue $q_{\mathrm{N}}$ : if $\Lambda[I]-\left(C_{\mathrm{C}}+\Lambda_{\mathrm{R}}[I]\right) \leq 0$, then step 14 .
Step 12: Occupation of the queue $q_{\mathrm{N}}$ with current value:

$Q_{\mathrm{N}}=\Lambda[I]-\Lambda_{\mathrm{R}}[I]-\left(N_{\mathrm{C}}+C_{\mathrm{C}}\right)+Q_{\mathrm{N}}$, assign the value for the number of unoccupied channels in the base station $C_{C}=N_{\text {C }}$.

Step 13: Check overflow for queue for data traffic for the current iteration: if $Q_{\mathrm{N}}>M_{\mathrm{N}}$, then the queue overflow for data traffic $b_{\mathrm{N}}=b_{\mathrm{N}}+1$, giving value for $Q_{\mathrm{N}}=M_{\mathrm{N}}$.

Step 14: Generation of the value for servicing of the busy channels in the base station with selected distribution $\Phi[1]$.

Step 15: Service employment channels in the base station. Determination of the number of outstanding unserved channels for the current iteration $\mathrm{Cc}$.

Step 16: Occupation of the free channel from 1 to $N_{\mathrm{N}}$ with data packets from the queue $q_{\mathrm{N}}$. Determination of the number of the busy channels with data packets $C_{\mathrm{N}}$ for the current iteration.

Step 17: Occupation of channels from $N$ to $N_{\mathrm{N}}+1$ and the rest unoccupied channels from $N_{\mathrm{N}}$ to 1 with voice calls in the queue $q_{\mathrm{R}}$.

Step 18: Set the next iteration $I=I+1$.

Step 19: When $(I+1)>n$, end of simulation, otherwise return to Step 3.

\section{Conclusions}

This paper discussed the problems of providing wireless access to a wide variety of multimedia services and applications, in parallel with ensuring the quality of service in modern cellular mobile networks.

A number of techniques for modeling and simulation of rare events are studied. The results show that the most effective are the methods Splitting and Importance Sampling. These techniques lead to major reductions in the ranges of variation of operating parameters, which requires the development of new approaches and methods for assessing the probability parameters of the QoS in broadband cellular networks.

In the present study an algorithm for determining the number of blocked calls in the channels of the base station and the number of overflows in the handover queues using a scheme with three priority is discussed. This algorithm is a stage of developed method that study rare events during signal transfer in broadband handover presented in [11].

\section{References}

1. Wang, J., Q. Zeng, Agrawal D. Member. Performance Analysis of a Preemptive and Priority Reservation Handoff Scheme for Integrated Service-Based Wireless Mobile Networks. - IEEE Transaction on Mobile Computing, 2, 2003, 65-75.

2. Zang, Y. Analysis of CSMA Based Broadcast Communication in Vehicular Networks with Hidden Stations. Ph. D. Dissertation, ABMT 75, 2015.

3. Georg, C. Stochastische Simulationstechnik mit Anwendungen auf Kommunikationsnetze, teil I+II, januar 2005, 6-8.

4. Maten, E., T. Doorn, J. Croon, A. Bargagli. Importance Sampling 
for High Speed, Statistical Monte-Carlo Simulations Handover Management Strategies. Eindhoven University of Technology, Department of Mathematics and Computer Science, CASA-Report 09-37, November, 2009, 2-64.

5. Heegaard, P. Efficient Simulation of Network Performance by Importance Sampling. Norwegian University of Science and Technology in Partial Fulfilment of the Requirements for the Degree of Doktor Ingeniwr, May, 1998.

6. Rubino, G., B. Tuffin. Rare Event Simulation Using Monte Carlo Methods. John Wiley \& Sons, 2009.

7. Siarova, S., D. Radev. Rare Event Simulation of Handover Priority Schemes for Broadband Wireless Networks. - Journal E+E, 46, 2011, 7-8, 34-42.

8. Radev, D., S. Radeva, I. Kurtev, D. Stankovski. Priority Handover Schemes in Wireless Mobile Networks. 18th Telecommunications forum TELFOR'10, Serbia, Belgrade, 2010, 320-323.

9. Otsetova-Dudin, E., S. Radeva, D. Radev. Modeling an Simulation of Handover Priority Schemes for Broadband Wireless Networks. IEEE 34-th International Conference ITUT'12, Croatia,

\section{Manuscript received on 02.11.2016}

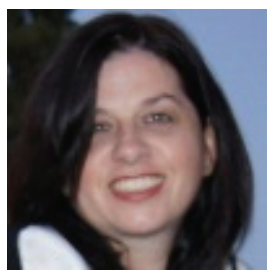

Assistant professor Ekaterina OtsetovaDudin was born in 1969 in Samokov. She received her high education as a MasterEngineer degree subject "Electronics and Automation" in the Technical University Sofia. She holds an academic degree PhD in Communication and Computer Technique. Ekaterina Otsetova-Dudin is assistant professor of Operating System, Software Technologies in Internet, Database at the University of Telecommunications and Post-Sofia. In addition to university teaching, Dr. Otsetova-Dudin has numerous publications and participation in $R \& D$ projects focused on telecommunications and computer simulations. She has more than 27 papers in Bulgarian and foreign Journal, one printed textbooks and five electronic courses for students. In parallel with his lecturer's activities from 2005 up to 2015, she worked as a administrator and head of the Center for Electronic and Distance Learning (University of Telecommunications and Post). Her area of scientific interest includes telecommunications and computer simulations.

Contacts:

Faculty of Telecommunications and Management University of Telecommunications and Post 1, Akad. St. Mladenov St., 1700 Sofia, Bulgaria e-mail: eotsetova@abv.bg

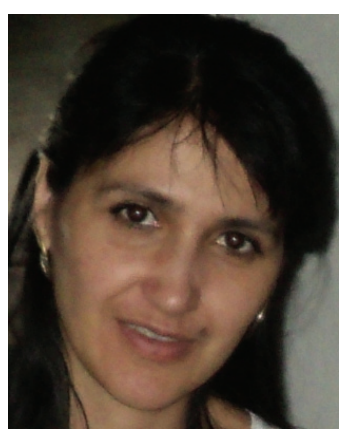

Svetlana Siarova born in 1969 in Ruse. She received her high education as a Master-Engineer degree subject "Electronics and Automation" in the Technical University - Sofia. She has more than six papers in Bulgarian and foreign Journal. Svetlana Siarova is a teacher in Sofia.

Contacts: e-mail:zvetlanas@yahoo.gr
2012, 449-454.

10. Radev, D., I. Kurtev, D. Stankovski, S. Siarova. A Handover Scheme for Broadband Wireless Mobile Networks. Proc. of International Symposium on Electronics and Telecommunications, ETC 2010, Timisoara, Romania, 2010, 137-140.

11. Otsetova-Dudin, E. Methodology for Simulation Study of Rare Events in Broadband Handover Realized with Priorities. Annual Proceeding of Higher School College of Telecommunications and Post, I, 2014, 5-9 (in Bulgarian).

12. L'Ecuyer, P., M. Mandjes, B. Tuffin. Importance Sampling in Rare Event Simulation. Book Chapter in Rare Event Simulation Using Monte Carlo Methods. John Wiley \& Sons, 2009, 17-38. 13. Radev, D. Rare Events Simulation in Broadband Digital Networks. Colbis. Sofia, 2006, 272-338 (in Bulgarian).

14. Otsetova-Dudin, E., S. Radeva, D. Radev. Modeling and Simulation of Handover Priority Schemes for Broadband Wireless Networks. ITI 2012, IEEE 34-th International Conference on Information Technology Interfaces, Zagreb, Croatia, 2012, 449-454.

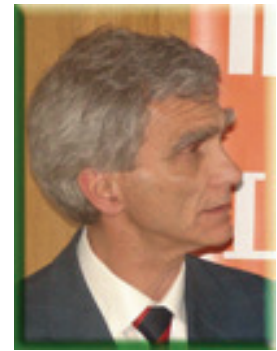

Prof. Ivan Kurtev was born in 1948 in Sofia. He received his high education as a Master-Engineer degree subject ,Electronics " in July 1973 and his Doctor's degree at the scientific subject ,Automation of the experiment" - Diploma HAC № 11089/ 30.07.1981, both in the Technical University - Sofia. He became an Associate Professor at the scientific subject „Computer Systems, Nets and Complex" - Diploma HAC № 10390/02.11.1987. Hi is Professor at scientific subject „Computer Systems, Nets and Complex“-Diploma №001 /10.06.2011 from the University of Telecommunications and Post-Sofia. From 2014 up to now hi is a part-time professor, lecturer of two courses in the field of computer sciences in the French Faculty of the Technical University - Sofia. Hi has more than 50 papers in Bulgarian and foreign journals, one patent, six printed textbooks and five electronic courses for students. In parallel with his lecturer's activities from 1976 up to now, he worked as a developper of computerised electronic devices during 32 years in the Central Institute of Computer Techniques - Sofia. His area of scientific interest includes application of microcomputers for industrial automation, telecommunications, measurements and electronic education and training.

Contacts:

French Education Faculty

Technical University of Sofia

8, Kl. Ohridski Blvd., 1000 Sofia, Bulgaria e-mail: i.kurtev@mail.bg 